\section{Oui ou non aux implants mammaires?}

\author{
E. Eicher
}

Le problème des implants mammaires, en particulier contenant ou enveloppés de silicone, a déjà une longue histoire. Au début des années 1990, un grand nombre de plaintes furent déposées par les porteuses d'implants qui souffraient d'indispositions de santé parfois graves. Des implants rompus, des fibroses capsulaires graves et la perturbation du système autoimmunitaire à cause de la silicone - fait encore non prouvé aujourd'hui - soulevèrent, depuis les EtatsUnis, une vague de protestation mondiale contre la pratique des implants au silicone. Les plaintes réunies des femmes américaines concernées mirent sous forte pression les producteurs d'implants. Certains des procès alors engagés sont encore en cours, bien que les entreprises aient essayé de calmer les esprits en présentant des comparaisons. Certains pays ont interdit les implants en silicone. Les patients et les associations d'entraide reprochèrent au corps médical suisse d'être nonchalant et passif et de se laisser aller à des manœuvres destinées à cacher ou à bagatelliser le problème. Les premiers contacts que les associations de médecins suisses prirent avec le SSF («Selbsthilfegruppe silikongeschädigter Frauen", groupe d'entraide silicone), démontrèrent à quel point les femmes concernées étaient inquiètes, non seulement à cause du matériau utilisé mais aussi par la façon dont les opérateurs qui posent les implants abordent le problème. Toute une série de reproches a été adressée à la FMH, sur l'information déficiente donnée aux patientes quant à l'intervention, sur la bagatellisation des éventuelles conséquences ultérieures, sur l'incompétence opérationnelle de certains chirurgiens, sur l'impuissance du corps médical lors de l'apparition de complications précoces ou tardives, le défaut d'information aux patientes sur l'implant utilisé, la numérotation et la description des produits, le refus de communiquer le compte-rendu d'opération, des modes de règlements incorrects et un comportement arrogant envers les patientes qui avaient à se plaindre des manquements précités.

Cette situation donna au Comité central le signal pour lancer une action, chercher des explications à tous ces reproches et faire des propositions pour améliorer la situation. Un groupe de travail a été consti-

Correspondance:

Dr Eduard Eicher

J. J. Balmerstrasse 10

CH-4053 Bâle
Groupe de travail Silicone

Eduard Eicher, Fédération des médecins suisses FMH (direction et modération)

André Aeschlimann, Société Suisse de Rhumatologie Urs Bösch, Société Suisse de Chirurgie plastique, reconstructive et esthétique

Renzo Brun del Re, Société Suisse de Gynécologie et d'Obstétrique

Ulrike Ehlers, Médecin assistante

Margrit Kessler, Organisation suisse des patients (OSP) Gaston-François Maillard, Société Suisse de Chirurgie plastique, reconstructive et esthétique, Société Suisse de Sénologie, European Committee on Quality Assurance of Medical Devices in Plastic Surgery (EQUAM)

Claudia Meuli-Simmen, Société Suisse de Chirurgie plastique, reconstructive et esthétique

Cosima Meyer, SSF Suisse (groupe d'entraide silicone)

Jan G. Poëll, Société Suisse de Chirurgie plastique, reconstructive et esthétique, European Committee on Quality Assurance of Medical Devices in Plastic Surgery (EQUAM)

Isabel Scuntaro, Office fédéral de la santé publique, Service Dispositifs médicaux

tué (voir cadre), avec la contribution des sociétés médicales spécialisées impliquées, des organisations de patients et de l'Office fédéral de la santé publique, sous la direction de l'auteur de cet article. Les premières discussions servirent à établir une liste des problèmes soulevés et à aplanir les aspects émotionnels des deux côtés. En tenant compte des nouvelles publications, il fut donc tenté d'élaborer une analyse aussi exacte que possible des différents implants avec ou sans silicone. Rapidement, les points suivants se sont dessinés pour les discussions suivantes:

1. améliorer l'information des femmes concernées;

2. examiner les possibilités d'enregistrement anonyme et complet des implants utilisés;

3. examiner et analyser scientifiquement les perturbations de santé pour les porteuses d'implants;

4. informer le public à différents niveaux, avec des standards européens de qualité, exigés pour les implants et les opérateurs;

5. réviser et rééditer la brochure éditée par l'Office fédéral de la santé publique «L'ABC des implants mammaires".

Cette brochure s'adresse aux femmes qui souhaitent un implant ou d'autres corrections de la poitrine, aux médecins traitants, à tous ceux qui sont confrontés à des complications éventuelles, aussitôt après l'intervention ou longtemps après, aux médecins qui se sont intéressés à la question des maladies de type rhumatismal susceptibles de survenir après l'implantation de silicone. La brochure a été entièrement révisée par le groupe de travail, et actualisée selon les dernières informations scientifiques. Sa mise en page a également été améliorée. 
La tâche du groupe de travail était fort complexe, et a absorbé beaucoup de temps. Elle semblait parfois une quête de la vérité, la recherche une ligne conductrice. A l'avenir, il sera important d'effectuer cette démarche pour les implémentations nécessaires. Personne ne devrait être opéré sans avoir reçu et lu ces explications dans le détail. Pour la diffusion de la brochure, le groupe de travail compte sur le soutien des sociétés médicales, qui doivent prendre leurs responsabilités du point de vue de l'amélioration de la qualité dans la problématique des implants au silicone. Les congrès annuels des sociétés médicales et les groupes de qualité locaux ont la possibilité de diffuser la brochure aux personnes opérées et au personnel médical qui s'occupe des traitements ultérieurs. Ce sont souvent les médecins de famille, qui sont confrontés avec les éventuelles complications et les difficultés que rencontrent les porteuses d'implants. Cette brochure peut être commandée gratuitement à l'adresse ci-dessous par les patientes et par les médecins. Une version existe sur Internet, complétée de différents liens avec d'autres sites. La brochure fournit de plus des renseignements sur les centres qui possèdent les compétences nécessaires pour aider les femmes en difficulté lors de complications. Il faut encore mentionner que différentes sociétés médicales ont, dans l'intervalle, déjà informé leurs membres sur la problématique posée par les implants mammaires, que ce soit lors de congrès ou grâce à leurs publications.

Il faut espérer que l'émotion bien compréhensible saura se calmer, que le thème des implants mammaires ne sera plus un tabou et que les femmes qui souffrent de complications recevront l'orientation adéquate vers l'aide nécessaire. Il ne faut cependant pas oublier que des milliers de femmes vivent sans problèmes avec un implant mammaire et sont très satisfaites de l'intervention subie. Le développement permanent des méthodes et des connaissances peut rendre nécessaire une nouvelle mise à jour de la brochure à bref délai.

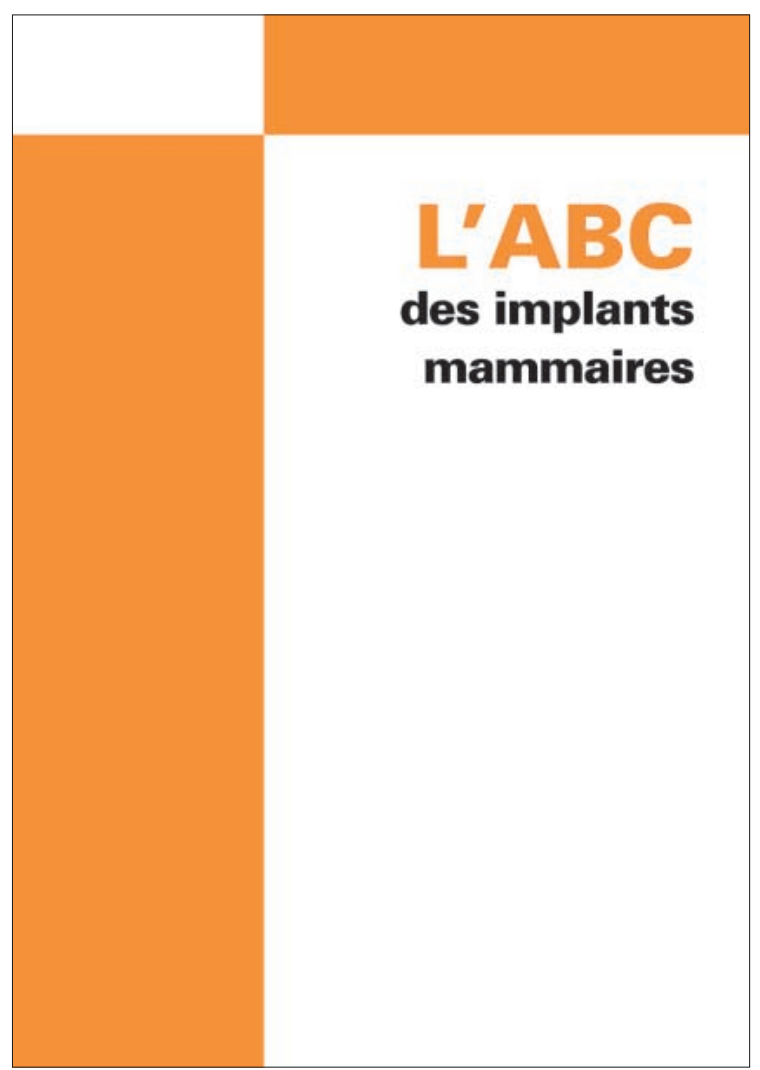

Des exemplaires imprimés de la brochure «ABC des implants mammaires" peuvent être retirés gratuitement auprès de l'OFCL/EDMZ 3003 Berne, numéro de commande $311.558 \mathrm{f}$.

La version Internet se trouve sous:

www.bag.admin.ch/md/f/abc.htm (en français), www.bag.admin.ch/md/d/abc.htm (en allemand), www.bag.admin.ch/md/i/abc.htm (en italien). 